\section{Vergleich von Immuntherapien beim NSCLC}

\begin{abstract}
Um die Wirkkraft von Checkpointinhibitoren zu erhöhen, könnte die Kombination mit einer weiteren Immuntherapie sinnvoll sein. Eine Metaanalyse liefert einen Überblick über den Stand der Immuntherapien beim NSCLC.
\end{abstract}

$E_{h}^{s}$ könnte sinnvoll sein, Checkpointinhibitoren mit weiteren immuntherapeutischen Strategien zu kombinieren, z. B. Vakzinierungen oder zellulären Therapien. Welche der beiden Optionen beim NSCLC am wirkungsvollsten ist, untersuchten Forscher in einer Metaanalyse. Sie werteten 18 randomisierte und kontrollierte Studien aus, die sie zur Therapie des NSCLC mit einem Tumorimpfstoff oder einer zellulären Therapie in den Datenbanken fanden. Ausgeschlossen waren Studien, in denen zudem Checkpointblocker oder Immunmodulatoren untersucht wurden. Insgesamt lagen Daten zu 6.756 Patienten vor. Die Verfahren waren zahlreich, darunter z. B. die Tumorimpfstoffe Tecemotide und MAGE-A3 sowie Therapien mit dendritischen Zellen und zytokininduzierten Killerzellen.

Den Daten zufolge verlängerte eine Immuntherapie progressionsfreies Überleben (PFS) und Gesamtüberleben (OS) gegenüber der jeweiligen Kontrollgruppe. Die Patienten im Kontrollarm erhielten je nach Design ein Placebo, eine Supportivbehandlung oder auch eine Chemotherapie (CT), sofern die Immuntherapie plus CT untersucht wurde. Die Hazard Ratio [HR] lag bei 0,81 für das OS $(\mathrm{p}=0,01)$ und bei 0,83 für das PFS $(\mathrm{p}=0,006)$. Das OS war um 5,43 Monate länger ( $p<0,005)$, das PFS um 3,24 Monate $(\mathrm{p}<0,005)$. Dabei erwiesen sich zelluläre Therapien als erfolgreicher als die Impfstoffe. Eine höhere Wirksamkeit bei Tumoren mit früherem Stadium und bei zeitgleicher CT zeigte sich nur hinsichtlich der Tumorprogression, nicht des OS. Es ergab sich kein signifikanter Effekt der Tumorhistologie auf PFS oder OS.

Fazit: Tumorimpfstoffe und zelluläre Immuntherapien verbesserten OS und PFS von NSCLC-Patienten. Die zelluläre Immuntherapie erwies sich dabei als effektiver als die Tumorimpfung. Eine zeitgleiche CT und ein frühes Krankheitsstadium verbesserten die Wirksamkeit - aber nur mit Blick auf wenige Krankheitsparameter.

Christina Berndt

Dammeijer F et al. The Efficacy of Tumor Vaccines and Cellular Immunotherapies in Non-Small Cell Lung Cancer: A Systematic Review and MetaAnalysis. J Clin Oncol. 2016;34(26):3204-12.

\title{
NSCLC: Raus, aber wie?
}

\section{Die Operationsmöglichkeiten beim NSCLC im Frühstadium sind vielfältig. Welche Methode ist wann die erfolgreichste - auch mit Blick auf die neue Unterteilung der T1a-Subgruppe?}

\begin{abstract}
n den letzten 20 Jahren galt die Lobektomie als operative Methode der Wahl für nichtkleinzellige Lungenkarzinome (NSCLC) im Stadium I $(\leq 3 \mathrm{~cm})$. Doch wie mit kleineren Tumoren $(\leq 2 \mathrm{~cm}) \mathrm{um}$ gegangen werden soll, ist bis heute unklar. Hinzu kommt, dass die Internatio-
\end{abstract}

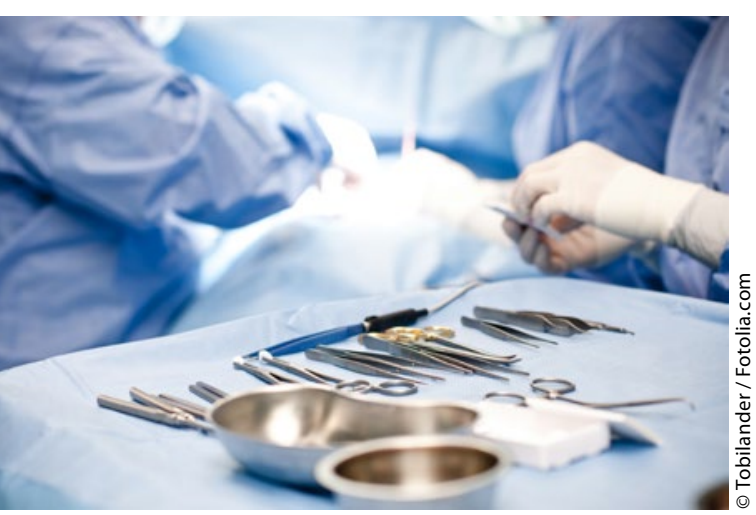

Eine Lobektomie ist wohl die beste Wahl bei frühen NSCLC-Stadien. nal Association for the Study of Lung Cancer (IASLC) erst vor kurzem Lungentumoren neu subklassifiziert hat. Die existierende Tla-Subgruppe $(\leq 2 \mathrm{~cm})$ wurde aufgeteilt in T1a $(\leq 1 \mathrm{~cm})$ und T1b (>1 bis $2 \mathrm{~cm}$ ), da sich die 5-Jahres-Überlebensraten der Patienten in diesen Gruppen sehr unterscheiden. Eine internationale Arbeitsgruppe hat deshalb die Operationsmöglichkeiten für diese beiden neuen Subgruppen mithilfe der Datenbank „Surveillance, Epidemiology, and End Results" (SEER) analysiert.

Insgesamt konnten Daten von 15.760 Patienten ausgewertet werden. Erfasst wurden das Gesamtüberleben (OS) und das Lungenkrebs-spezifische Überleben (LCSS) nach Lobektomie, Segmentektomie und Wedge-Resektion. OS und LCSS waren nach einer Lobektomie am günstigsten - für Patienen mit Tla-NSCLC sowie für solche mit T1b-NSCLC. Eine multivariate Analyse zeigte, dass die Segmentektomie und die Wedge-Resektion unabhängig mit einem kürzeren OS und LCSS assoziiert waren als die Lobektomie. Bei T1b-Tumoren ergaben sich nach Wedge-Resektion im Vergleich zur Segmentektomie ungünstigere Ergebnisse hinsichtlich OS und LCSS, während für Tumoren $\leq 1 \mathrm{~cm}$ mit beiden Methoden ähnliche Resultate erzielt wurden. Multivariate Analysen zeigten hier, dass die Wedge-Resektion ein unabhängiger Risikofaktor für das OS bei Tumoren $>1 \mathrm{~cm}$ ist, aber nicht bei Tumoren $\leq 1 \mathrm{~cm}$.

Fazit: Nach Lobektomie ergaben sich für Patienten mit NSCLC-Tumoren $\leq 1 \mathrm{~cm}$ sowie mit Tumoren $>1$ bis $2 \mathrm{~cm}$ bessere Überlebenschancen als nach sublobarer Resektion. Patienten, bei denen eine Lobektomie nicht möglich ist, sollte eine Segementektomie empfohlen werden, sofern ein T1b-NSCLC vorliegt. Dagegen können Chirurgen bei einem NSCLC $\leq 1 \mathrm{~cm}$ mit Blick auf ihre operativen Fertigkeiten und das Patientenprofil zwischen Segmentektomie und WedgeResektion wählen.

Christina Berndt

Dai $C$ et al. Choice of Surgical Procedure for Patients With NSCLC $\leq 1 \mathrm{~cm}$ or $>1$ to $2 \mathrm{~cm}$ Among Lobectomy, Segmentectomy, and Wedge Resection: A Population-Based Study. J Clin Oncol. 2016;34(26):3175-82. 